\title{
Particle Focusing and Separation through Lab-On-Chip Device by Dielectrophoresis
}

\author{
Deli Liu ${ }^{*}, 1$, Baoyu Song ${ }^{1}$, Liguo Chen ${ }^{1,2}$ and Lining Sun ${ }^{1,2}$ \\ ${ }^{I}$ State Key Laboratory of Robotics and System, Harbin Institute of Technology, Harbin 150080, China \\ ${ }^{2}$ Robotics and Microsystems Center, Soochow University, Suzhou, 215021, China
}

\begin{abstract}
A Lab-On-Chip micro-device has been introduced for the focusing and separation of particles which exploits negative dielectrophoretic (nDEP) force. A 3D numerical computational model was presented to simulate the focusing and separation process. In particular, the model will solve for bioparticle movement and Joule heating. In the experimental study, the mixture of viable and nonviable yeast cells was used to demonstrate the focusing and separation effect of the designed microfluidic device. The fabricated chip performs a desired focusing result for the conditions with the electric fields frequency of $1 \mathrm{MHz}$, the medium conductivity of $0.01 \mathrm{~S} / \mathrm{m}$ and the applied voltage $V=20 V_{p p}$. The mixture of viable and nonviable yeast cells were focused to the central plane of the micro-channel and then separated by non-equilibrium nDEP induced by the single part of the last pair of electrodes with conductivity $\sigma=0.01 \mathrm{~S} / \mathrm{m}$, applied voltage $V=20 V_{p p}$ and applied electric field frequency of $5 \mathrm{Mhz}$, which validities the prediction of the proposed 3D model. This Lab-On-Chip device provides a simple and effective mean of particle focusing.
\end{abstract}

Keywords: Lab-On-Chip, dielectrophoresis, particle focusing, particle separation.

\section{INTRODUCTION}

The recent development of bio-microfluidic devices makes it possible to perform complex functions of biological assays on a Lab-On-Chip device. Lower cost, lower volumes of sample and shorter time are considered to be the advantages of bio-microfluidic devices. For typical biomedical applications, the focusing of cells from dilute suspension or even a targeted location is desired using a bio-microfluidic device. Many focusing techniques have been developed to accomplish the focusing of targeted particles through sheath flow confine the particles within a certain volume of fluid stream. These methods are effective for focusing and later on for separation but the device are complex and require multiple inlets with precise flow rates. Due to these constraints the device is difficult to be fabricated and sometimes cannot be used commercially due to its cost effectiveness. Dielectrophoresis provides an attractive alternative to conventional separation and focusing methods because of its ability to separate bioparticles in a convenient, controllable, selective and rapid means $[1,2]$. Braschler et al. proposed a multiple frequencies dielectrophoresis method to separate cells continuously [3]. They produced electric field gradients by a novel geometrical arrangement of lateral metal electrodes and a patterned insulator. And the performance of this device was demonstrated by separating a mixed yeast cell population into pure fractions of viable and nonviable cells. In addition, red blood cells infected with Babesia bovis were enriched by using the same device which confirms the hypothesis that infection with $\mathrm{B}$. bovis causes significant changes in the dielectric response of red blood cells. The

*Address correspondence to this author at the School of Mechanical Engineering, Soochow University, Suzhou, 215021, China; Tel: +8613771766879; E-mail: drliudeli@gmail.com proposed geometrical arrangement of the lateral dielectrophoresis or "liquid electrodes" is characterized and optimized for shaping electric field to achieve lateral deviation of particles in liquid flow.

DEP means the creation of forces on polarizable particles and the resulting movement of them in a nonuniform electric field. The magnitude and direction of DEP forces depend on the frequency of the AC electric field, conductivity and permittivity of both particles and the medium where particles suspended, and the gradient of electric field. Confining the sample to the center of the microfluidic channel ensures that all the particles move at a constant velocity. It also eliminates signal interference from the chamber wall, as well as particle-wall interactions [4]. Chu et al. proposed a microfluidic device using lateral dielectrophoresis to focus and continuously separate cells [5].

In this work, a 3D computational model was presented to predict and investigate the particle behavior and the impact of Joule heating. The present microfluidic device, similar to that of Demierre et al., can focus particles eliminating the use of sheath flow and pumps. Experiments were conducted to verify the proposed $3 \mathrm{D}$ computational model.

\section{NUMERICAL MODEL AND GOVERNING EQUATIONS}

Microfluidic devices often have complex electrode patterns, which require solving the full set of governing equations using numerical method. Comsol Multiphysics was used to simulate the effects of temperature distribution on AC electrothermal microflow. Three modules are used to find the electric field, temperature distribution and fluidic velocity. First, the electric field distribution in the fluidic chamber is derived. The resulting electric field distribution is 


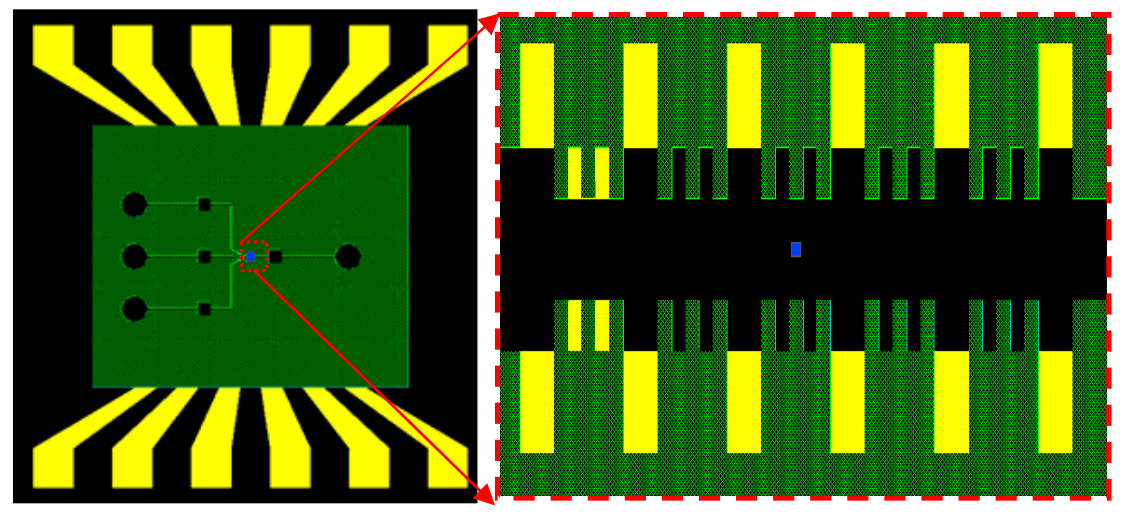

\section{Scale bar $100 \mu \mathrm{m}$}

Fig. (1). The schematic of the lateral microfluidic microelectrodes structure.

used to calculate the temperature field according to the energy equation. Then the fluid volume force in the chamber is calculated using the temperature gradient and the electric field distribution from the first two steps. Lastly, the fluid flow field is obtained by Navier-Stokes equation. The problem space and boundary conditions for the electrical and thermal problem are modeled similarly to the work by Green et al. [6], and the lateral microfluidic microelectrodes structure is shown schematically in Fig. (1).

The thermal and electrical problems are decoupled since induced fluid convection does not contribute to the thermal problem. The electrical problem is bounded to within the fluid medium. The Laplace equation for a potential $(\phi)$ in a homogeneous medium is first solved with [6].

$$
\nabla^{2} \phi=0, \quad \vec{E}=-\nabla \phi
$$

where the boundary condition for the electrode strip $V_{0}$ refers to the root mean square (rms) value of the applied potential.

The electrical force per unit volume for an incompressible fluid can be expressed as:

$$
\vec{F}_{e t}=-\frac{1}{2}\left[\left(\frac{\nabla \sigma}{\sigma}-\frac{\nabla \varepsilon}{\varepsilon}\right) \vec{E} \frac{\varepsilon \vec{E}}{1+(\omega \tau)^{2}}+\frac{1}{2}|\vec{E}|^{2} \nabla \varepsilon\right]
$$

The gradients in conductivity and permittivity can be replaced by corresponding gradients in temperature for aqueous buffer near room temperature

$$
\frac{\nabla \sigma}{\sigma}=0.002 \nabla T ; \frac{\nabla \varepsilon}{\varepsilon}=-0.004 \nabla T
$$

Then, the fluid velocity, $u$, is solved for using NavierStokes equation.

$-\nabla p+\eta \nabla^{2} u+\vec{F}_{e t}=0$

where $p$ is pressure, $\eta$ is fluid viscosity. The water properties are given as $\rho=1,000 \mathrm{~kg} / \mathrm{m}^{3}$ and $\eta=0.001 \mathrm{pa} \cdot \mathrm{s}$ at $20^{\circ} \mathrm{C}$. No slip boundary condition, $u=0$ is applied to all the solid boundaries.

The time averaged DEP force is given by [7]

$$
\left\langle\bar{F}_{D E P}(t)\right\rangle=2 \pi \varepsilon_{m} r^{3} \operatorname{Re}[K(\omega)] \nabla\left|\bar{E}_{r m s}\right|^{2}
$$

where $\varepsilon_{m}$ is the medium permittivity, $\nabla\left|\bar{E}_{r m s}\right|^{2}$ is the gradient of the square of rms electrical field, $\omega$ is the angular field frequency, $\operatorname{Re}[K(\omega)]$ is the real part of $K(\omega)$. $\operatorname{Re}[K(\omega)]>0$ means that cells show pDEP response while $\operatorname{Re}[K(\omega)]<0$ means that $\mathrm{nDEP}$ response.

Numerical simulations were conducted for different experimental fluid conductivities and applied voltage. Fig. (2) shows the FEM simulation of the particle tracing of yeast cells for a fluid sample with conductivity $\sigma=0.01 \mathrm{~S} / \mathrm{m}$, applied voltage $V=20 V_{p p}$, and electric field frequency of $1 \mathrm{MHz}$. From simulation results it can be seen that evenly distributed yeast cells in the inlet channel were focused to the central plane of the channel. Both viable (green lines) and nonviable yeast cells (red lines) present a similar focusing effect, and their stream lines overlapped when yeast cell pass a certain distance (here four electrodes gap). Fig. (3) shows FEM simulation result for the separation process of viable and nonviable yeast cells. $5 \mathrm{MHz}$ electric field frequency was applied at the single part of the last pair of electrodes to obtain the spatial separation of viable and nonviable yeast cells mixture with conductivity $\sigma=0.01 \mathrm{~S} / \mathrm{m}$ and applied voltage $V=20 V_{p p}$. Viable and nonviable yeast cells demonstrate an obviously distinctive stream line due to the different nDEP response. Strong electric field will act on the conducting fluid medium, which may result in Joule heating. This could damage temperature sensitive biological samples. It is important to monitor the temperature field in a fluid medium. Fig. (4) shows FEM simulation of the temperature field for a fluid sample with conductivity $\sigma=$ $0.01 \mathrm{~S} / \mathrm{m}$ and applied voltage $V=20 V_{p p}$. The maximum temperature increase from numerical simulation was less than $0.5^{\circ} \mathrm{C}$, which is safe for the yeast cells.

\section{DEVICE FABRICATION}

Device fabrication involves two parts, the electrodes and the microfluidic channel. First, the electrode array with 


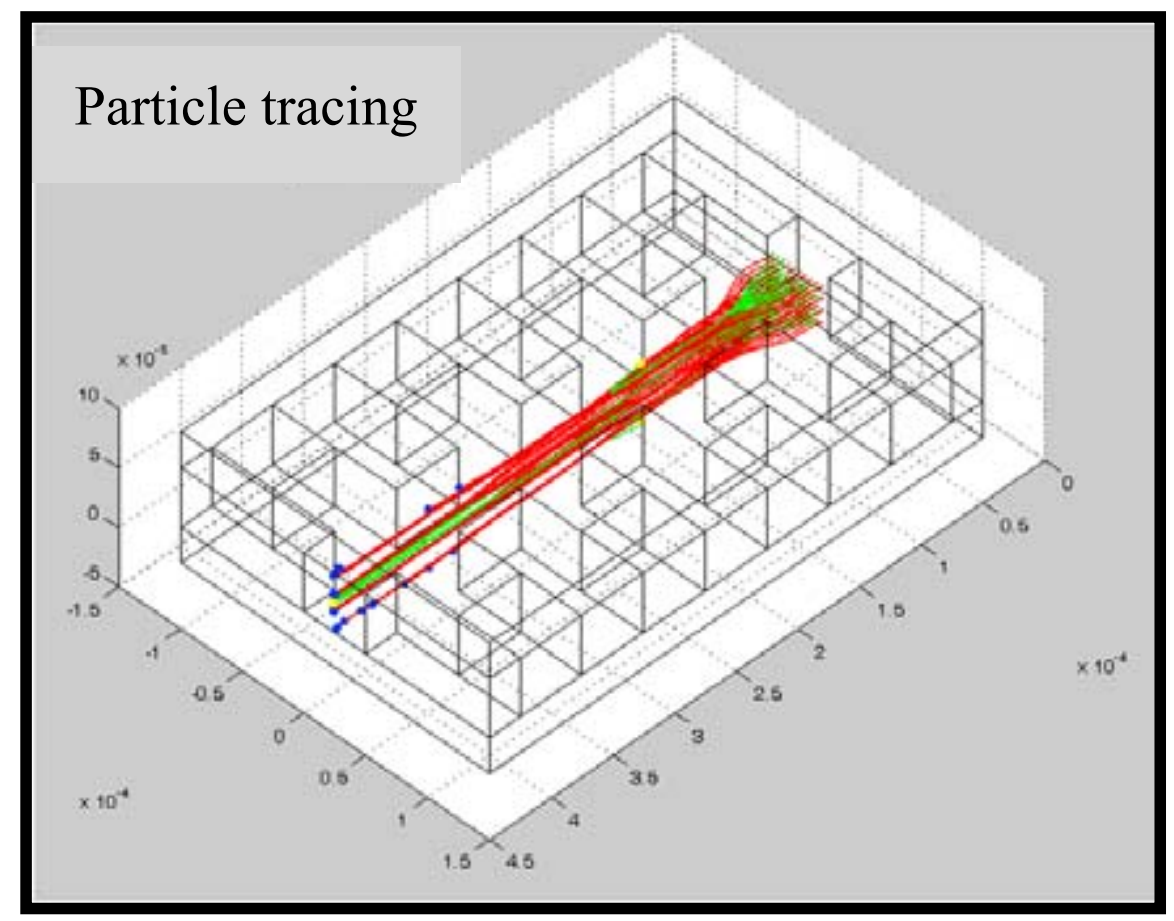

Fig. (2). FEM simulation of the particle tracing of yeast cells for a fluid sample with conductivity $\sigma=0.01 \mathrm{~S} / \mathrm{m}$ and applied voltage $V=$ $20 V_{p p}$.

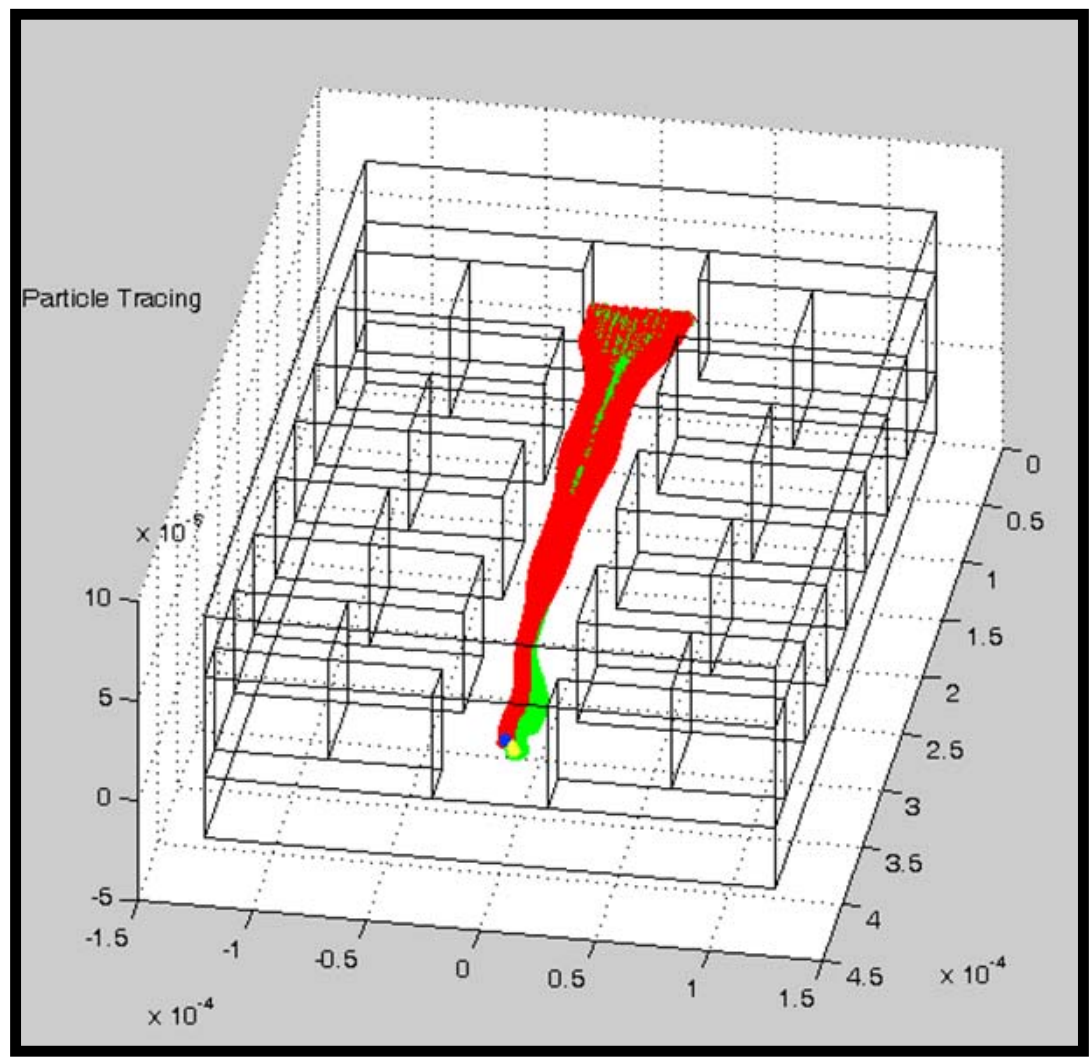

Fig. (3). FEM simulation of the separation process viable and nonviable of yeast cells for a fluid sample with conductivity $\sigma=0.01 \mathrm{~S} / \mathrm{m}$, applied voltage $V=20 V_{p p}$ and frequency $5 \mathrm{Mhz}$.

$50 \mu m$ wide, $50 \mu m$ spaced, were fabricated from Indium Tin Oxide (ITO) coated glass slide (SPI). These glass slides provide high transparency and good electrical conductivity, enabling visualization of the flow of particle in the microchannel. The chambers and the channels were structured in an insulating material. The microfluidic channels were made 


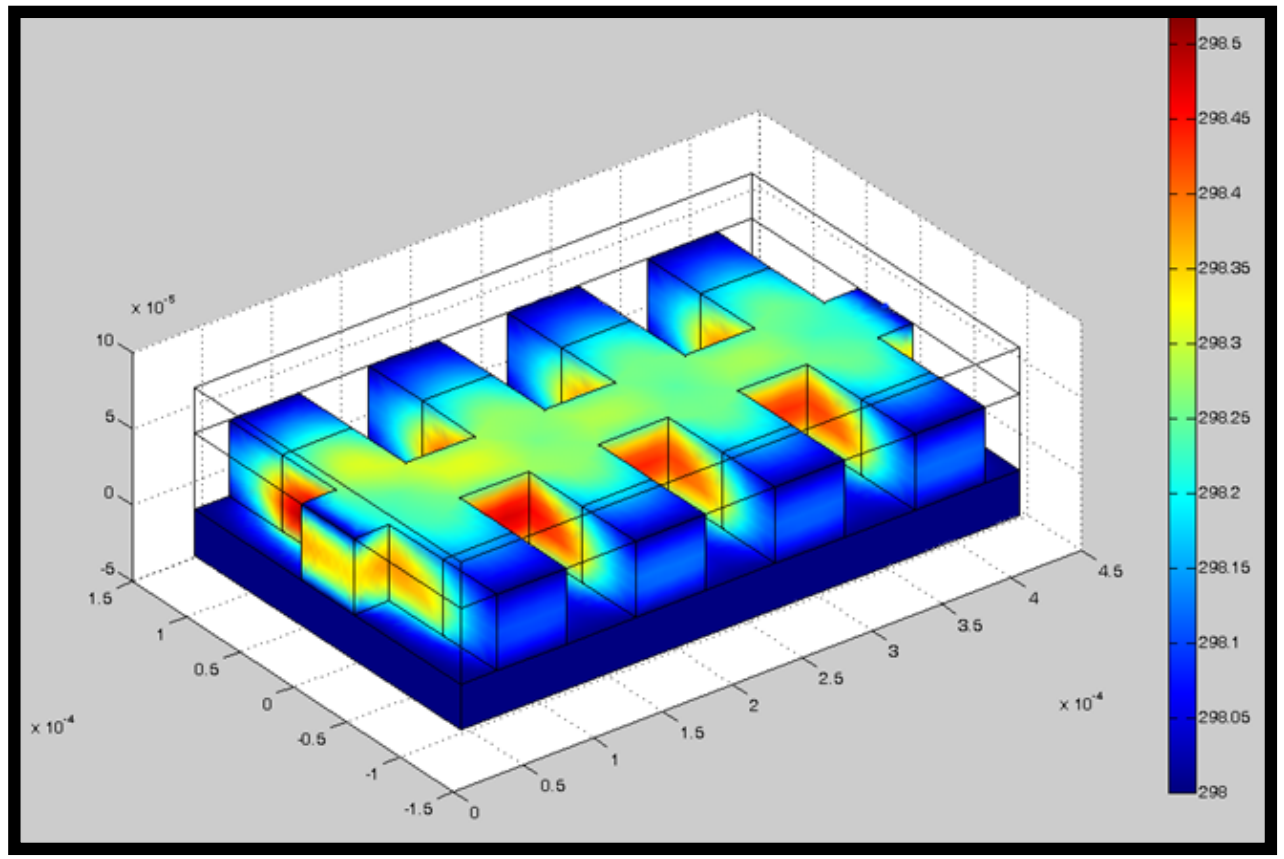

Fig. (4). FEM simulation of the temperature field for a fluid sample with conductivity $\sigma=0.01 \mathrm{~S} / \mathrm{m}$ and applied voltage $V=20 V_{p p}$.
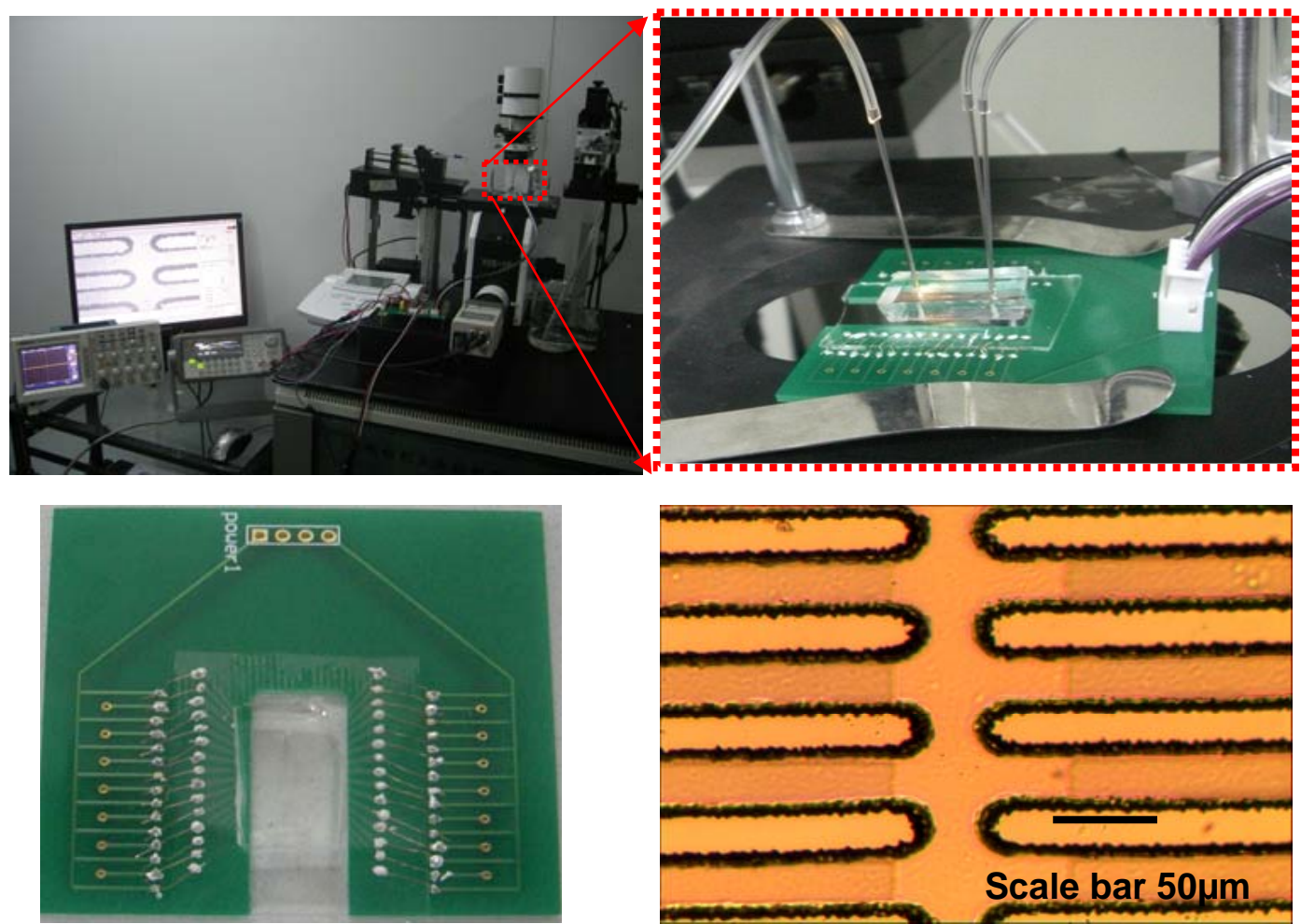

Fig. (5). The structure of the designed microfluidic chip system.

from PDMS structures that patterned on the bottom ITO glass substrate by standard photolithography process. The bottom-patterned structure was accurately positioned under a mask aligner. More details can be found in reference [7]. The structure of the designed microfluidic chip system is shown in Fig. (5). There are totally fifteen pairs of electrodes were fabricated on the bottom of the prototype microfluidic chip.

\section{TEST METHOD AND RESULTS}

The mixture of viable (live) and nonviable (dead) yeast (Sacchararomyces cerevisiae) cells was selected to serve as sample cells to be focused. Yeast cell are cultured in culture medium at $30^{\circ} \mathrm{C}$ for $24 \mathrm{~h}$, washed and then resuspended four times through deionized water. Conductivity of the medium is adjusted by adding a small amount of $\mathrm{NaCl}$ and conduc- 


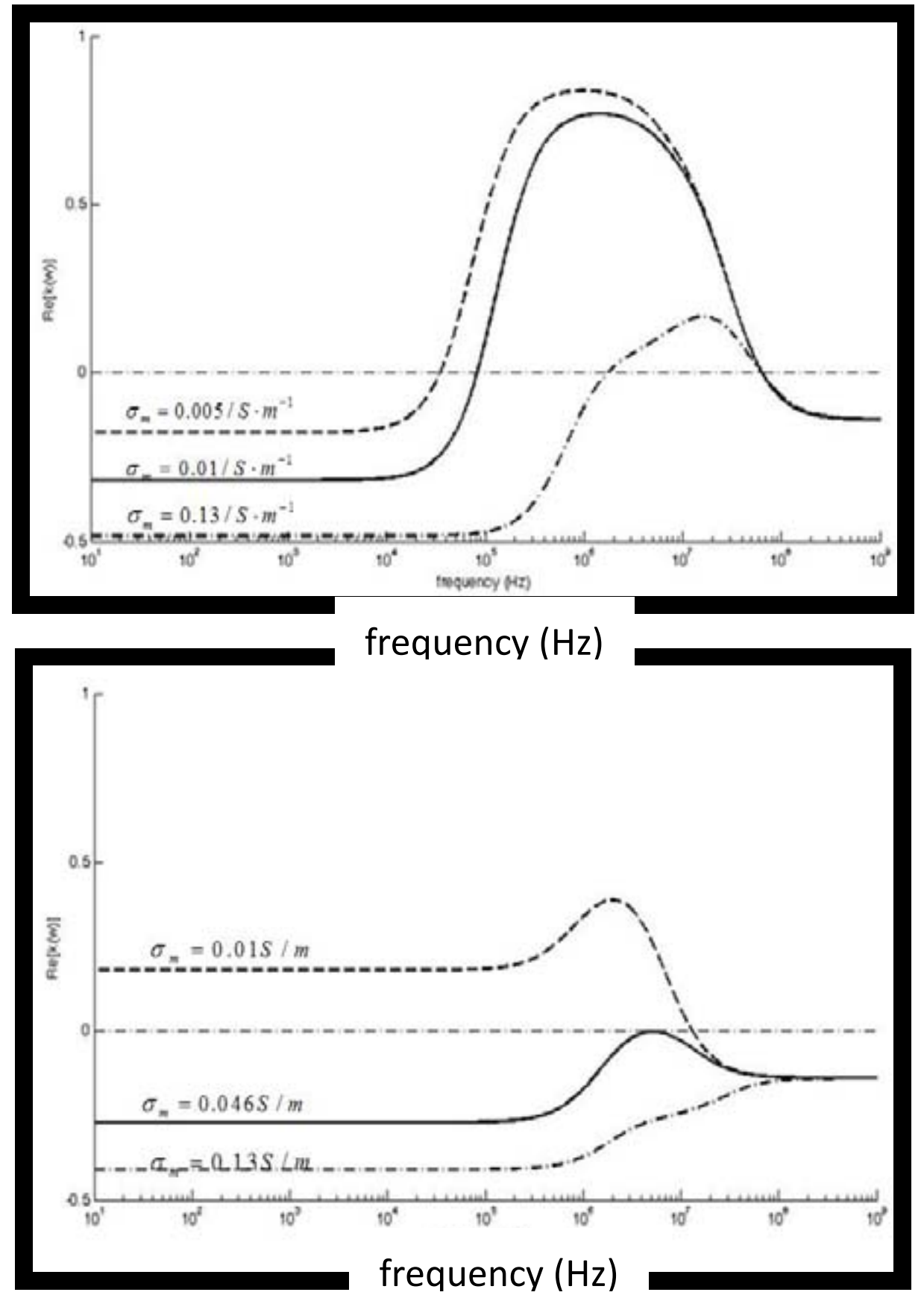

Fig. (6). The DEP frequency response at three different medium conductivities of viable (Upper) and nonviable (Lower).

tivity is measured by conductive meter TN2301. Nonviable yeast cells are prepared by heat treatment $\left(90^{\circ} \mathrm{C}\right.$ for $\left.20 \mathrm{~min}\right)$ and viability is visualized using a methylene blue stain. In this stain, the color of those dead cells changes into blue. Such a sample preparation follows the process proposed by Doh et al. [8]. In order to obtain the optimal condition of separation; we measure DEP response of viable and nonviable yeast cells using test devices in terms of the medium conductivity and the electric fields frequency before the particles focusing experiment. The results of DEP frequency response at three different medium conductivities of viable and nonviable were shown in Fig. (6).

The measurements using the mixture of viable and nonviable yeast cells were conducted to verify their focusing effect. The medium conductivity of $0.01 \mathrm{~S} / \mathrm{m}$ and electric frequency of $1 \mathrm{MHz}$ were chosen as the focusing condition because viable and nonviable yeast cells show same nDEP response at this conditions and low medium conductivity is good to avoid heat problem which can damage cells. Under these conditions, the mixture of viable and nonviable yeast cells was focused towards the center line across the channel, as shown in Fig. (7). The focusing effect was obtained by generating two opposite negative DEP-forces with the same low frequency signals. In order to separate the viable and nonviable yeast cell mixture from the focused fluid medium, a $5 \mathrm{MHz}$ electric field frequency was applied at the single part of the last pair of electrodes to obtain the spatial separation of viable and nonviable yeast cells mixture with 


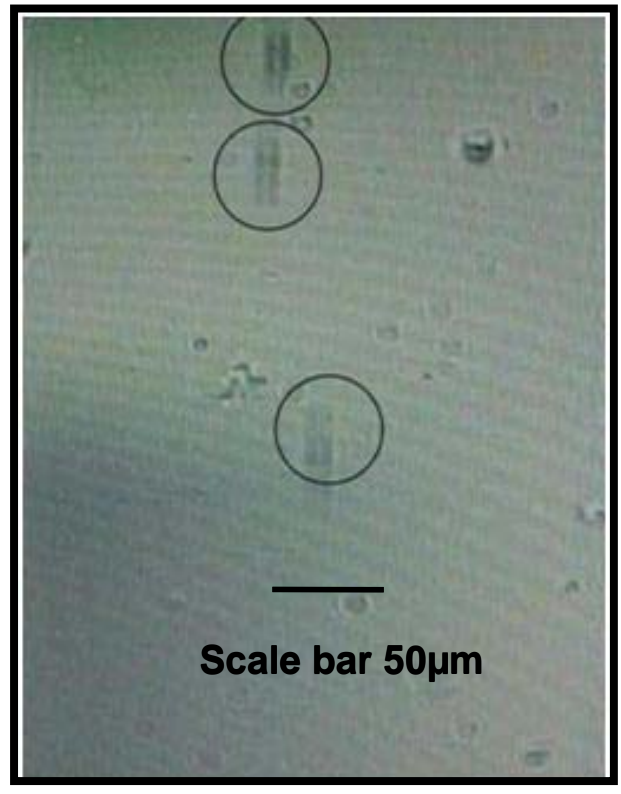

Fig. (7). The DEP focusing result of the mixture of viable and nonviable yeast cells with conductivity $\sigma=0.01 \mathrm{~S} / \mathrm{m}$ and applied voltage $V=20 V_{p p}$.

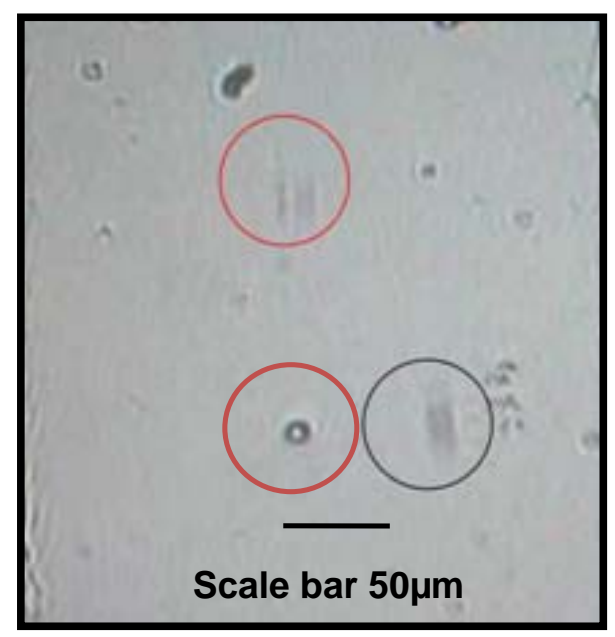

Fig. (8). The DEP separation result of the mixture of viable (indicated by the red circle) and nonviable yeast cells (indicated by the black circle) with conductivity $\sigma=0.01 \mathrm{~S} / \mathrm{m}$ and applied voltage $V=20 V_{p p}$.

conductivity $\sigma=0.01 \mathrm{~S} / \mathrm{m}$ and applied voltage $V=20 V_{p p}$. Fig. (8) shows the separation effect of viable (indicated by the red circle) and nonviable yeast cell (indicated by the black circle) which caused by the different nDEP response. There is a qualitatively agreement between the FEM simulation results and the experimental test results.

\section{CONCLUSIONS}

In this work, we present a cell focusing and separation microfluidic chip using negative dielectrophoresis. A 3D computational model was proposed to predict and investigate the behavior of particles. In the experimental study, viable and nonviable yeast cells were chosen to verify the focusing and separation effect of the proposed microfluidic chip. The mixture of viable and nonviable yeast cells were focused to the central plane of the micro-channel with conductivity $\sigma=$ $0.01 \mathrm{~S} / \mathrm{m}$ and applied voltage $V=20 V_{p p}$, and then separated by non-equilibrium nDEP induced by the single part of the last pair of electrodes which is in accordance with the prediction of the proposed $3 \mathrm{D}$ model. The present chip is capable of performing the work of hydrodynamic focusing and separation, and is promising to be integrated into biological analysis system.

\section{ACKNOWLEDGMENTS}

The authors acknowledge funding of the National Natural Science Foundation of China under Grant No. 60605025 and the National High Technology Research and Development Program of China (863 Program) under Grant No. 2009AA043703.

\section{REFERENCES}

[1] H. Pohl, "The motion and precipitation of suspensoids in divergent electric fields", Appl. Phys. (Berl), vol. 22, pp. 869-871, 1951.

[2] E. Cummings, and A. Singh, "Dielectrophoresis in microchips containing arrays of insulating posts: theoretical and experimental results", Anal. Chem., vol. 75, pp. 4724-4731, 2003.

[3] T. Braschler, N. Demierre, E. Nascimento, T. Silva, A. G. Oliva, and P. Renaud, "Continuous separation of cells by balanced dielectrophoretic forces at multiple frequencies", Lab. Chip., vol. 8, pp. 280-286, 2008.

[4] H. Morgan, D. Holmes, and N. G. Green, "3D focusing of nanoparticles in microfluidic channels", IEE Proc. Nanobiotechnol., vol. 150, pp. 76-81, 2003.

[5] N. Demierre, T. Braschler, R. Muller, and P. Renaud, "Focusing and continuous separation of cells in a microfluidic device using lateral dielectrophoresis", Sensors Actuat., vol. 132, pp. 388-396, 2008.

[6] N. G. Green, A. Ramos, A. Gonazalez, A. Castellanos, and H. Morgan, "Electric field induced fluid flow on microelectrodes: The effect of illumination", J. Phys. D: Appl. Phys., vol. 33, pp. L13L17, 2000.

[7] H. H. Cui, J. Voldman, X. F. He, and K. M. Lim, "Separation of particles by pulsed dielectrophoresis", Lab. Chip, vol. 9, pp. 23062312, 2009.

[8] Doh and Y. Cho, "A continuous cell separation chip using hydrodynamic dielectrophoresis (DEP) process", Sensors Actuat. A: Phys., vol. 121, pp. 59-65, 2005. 